\title{
Production of $X(3872)$ accompanied by a soft pion at hadron colliders
}

\author{
Eric Braaten, ${ }^{*}$ Li-Ping He $\odot,{ }^{\dagger}$ and Kevin Ingles ${ }^{*}$ \\ Department of Physics, The Ohio State University, Columbus, Ohio 43210, USA
}

(Received 29 March 2019; published 12 November 2019)

\begin{abstract}
If the $X(3872)$ is a weakly bound charm-meson molecule, it can be produced by the creation of $D^{* 0} \bar{D}^{0}$ or $D^{0} \bar{D}^{* 0}$ at short distances followed by the formation of the bound state from the charm-meson pair. The $X$ can also be produced by the creation of $D^{*} \bar{D}^{*}$ at short distances followed by the rescattering of the charmmeson pair into $X \pi$. At a high-energy hadron collider, the prompt cross section from this mechanism has a narrow peak in the $X \pi$ invariant mass distribution near the $D^{*} \bar{D}^{*}$ threshold from a charm-meson triangle singularity. An order-of-magnitude estimate of the ratio of the cross section for producing the peak in the $X \pi^{ \pm}$distribution to the cross section for producing $X$ without an accompanying pion suggests that the peak may be observable at the LHC.
\end{abstract}

DOI: 10.1103/PhysRevD.100.094006

\section{INTRODUCTION}

The discovery of a large number of exotic hadrons containing a heavy quark and its antiquark presents a major challenge to our understanding of QCD [1-10]. The first of these exotic hadrons to be discovered was the $X(3872)$ meson: it was discovered in 2003 in exclusive decays of $B^{ \pm}$ mesons into $K^{ \pm} X$ through the decay of $X$ into $J / \psi \pi^{+} \pi^{-}$ [11]. Its existence was quickly verified through inclusive production in $p \bar{p}$ collisions [12]. The $J^{P C}$ quantum numbers of $X$ were eventually determined to be $1^{++}$ [13]. Its mass is extremely close to the $D^{* 0} \bar{D}^{0}$ threshold, with the difference being only $0.01 \pm 0.18 \mathrm{MeV}$ [14]. This suggests that $X$ is a weakly bound $\mathrm{S}$-wave charm-meson molecule with the flavor structure $[15,16]$

$$
|X(3872)\rangle=\frac{1}{\sqrt{2}}\left(\left|D^{* 0} \bar{D}^{0}\right\rangle+\left|D^{0} \bar{D}^{* 0}\right\rangle\right) .
$$

However, there are alternative models for the $X$ [1-10]. The $X$ has been observed in many more decay modes than any of the other exotic heavy hadrons. In addition to the discovery mode $J / \psi \pi^{+} \pi^{-}$, it has been observed in $J / \psi \pi^{+} \pi^{-} \pi^{0}, J / \psi \gamma, \psi(2 S) \gamma, D^{0} \bar{D}^{0} \pi^{0}, D^{0} \bar{D}^{0} \gamma$, and most recently $\chi_{c 1} \pi^{0}$ [17]. The observation of $X$ in these 7 different decay modes has not proven to be effective in

\footnotetext{
*braaten.1@osu.edu

†he.1011@buckeyemail.osu.edu

*ingles.27@buckeyemail.osu.edu
}

Published by the American Physical Society under the terms of the Creative Commons Attribution 4.0 International license. Further distribution of this work must maintain attribution to the author(s) and the published article's title, journal citation, and DOI. Funded by SCOAP ${ }^{3}$. discriminating between these models. There may be aspects of the production of $X$ that are more effective at discriminating between models than the decays of $X$.

The $X$ can be produced by any reaction that can produce its constituents $D^{* 0} \bar{D}^{0}$ and $D^{0} \bar{D}^{* 0}$. In particular, it can be produced in high energy hadron collisions. The inclusive production of $X$ in $p \bar{p}$ collisions has been studied at the Tevatron by the CDF [12] and D0 [18] collaborations. The inclusive production of $X$ in $p p$ collisions has been studied at the Large Hadron Collider (LHC) by the LHCb [19], CMS [20], and ATLAS [21] collaborations. At a highenergy hadron collider, $X$ is produced by the weak decays of bottom hadrons, and it is also produced promptly through QCD mechanisms that create charm quarks and antiquarks. The substantial prompt production rate of $X$ at hadron colliders has often been used as an argument against its identification as a weakly bound charm-meson molecule. This argument is based on an upper bound on the cross section for producing $X$ in terms of the cross section for producing the charm-meson pair $D^{* 0} \bar{D}^{0}$ integrated up to a maximum relative momentum $k_{\max }$ [22]. The estimate for $k_{\max }$ in Ref. [22] was approximately the binding momentum $\gamma_{X}$ of the $X$. In Ref. [23], it was pointed out that the derivation of the upper bound in Ref. [22] requires $k_{\max }$ to be of the order of the pion mass $m_{\pi}$ instead of $\gamma_{X}$. In Ref. [24], we used the methods of Ref. [23] to derive an estimate for the $X$ cross section. The estimates for the prompt cross sections for $X$ at the Tevatron and at the LHC are compatible with experimental lower bounds on the cross sections.

The production of $X$ can also proceed through the creation of a pair of spin-1 charm mesons $D^{*} \bar{D}^{*}$ at short distances followed by their rescattering into $X \pi$. In Ref. [25], we studied the decays of $B$ mesons into $K X \pi$ 
from the decay at short distances into $K$ plus a $D^{*} \bar{D}^{*}$ pair followed by the rescattering of the charm-meson pair into $X \pi$. We used a previous isospin analysis of the decays $B \rightarrow K D^{(*)} \bar{D}^{(*)}$ [26] to estimate the short-distance amplitudes for creating the $D^{*} \bar{D}^{*}$ pair. We used an effective field theory called XEFT [27] to calculate the amplitudes for the rescattering of $D^{*} \bar{D}^{*}$ to $X \pi$ with small relative momentum. The differential decay rate has a narrow peak in the $X \pi$ invariant mass near the $D^{*} \bar{D}^{*}$ threshold from a charmmeson triangle singularity. We obtained an estimate of the branching fraction into the peak from the charm-meson triangle singularity in the decays $B^{0} \rightarrow K^{+} X \pi^{-}$and $B^{+} \rightarrow$ $K^{0} X \pi^{+}[25]$.

In this paper, we study the inclusive prompt production of $X \pi$ from rescattering of $D^{*} \bar{D}^{*}$ in high energy hadron collisions. The outline of this paper is as follows. In Sec. II, we describe some universal aspects of weakly bound $\mathrm{S}$-wave molecules as well as the effective field theory XEFT for charm mesons and pions. In the subsequent sections, we apply XEFT to various cross sections at a high-energy hadron collider. We consider the production of $X$ in Sec. III and the production of a pair of spin-1 charm mesons in Sec. IV. In Sec. V, we calculate the cross section for producing $X \pi$ with invariant mass near the $D^{*} \bar{D}$ threshold. We summarize our results and discuss their implications in Sec. VI.

\section{WEAKLY BOUND S-WAVE MOLECULE AND XEFT}

If short-range interactions produce an S-wave bound state extremely close to a scattering threshold, the few-body physics has universal aspects that are determined by the binding momentum $\gamma_{X}$ of the bound state [28]. The binding energy is $\gamma_{X}^{2} /(2 \mu)$, where $\mu$ is the reduced mass of the constituents. The momentum-space wave function in the region of relative momentum $k$ below the inverse range has the universal form

$$
\psi_{X}(k)=\frac{\sqrt{8 \pi \gamma_{X}}}{k^{2}+\gamma_{X}^{2}} .
$$

The low-energy scattering of the constituents also has universal aspects determined by $\gamma_{X}$ through a simple function of the complex energy $E$ relative to the scattering threshold:

$$
f_{X}(E)=\frac{1}{-\gamma_{X}+\sqrt{-2 \mu E}} .
$$

The universal elastic scattering amplitude in the region of relative momentum $k$ below the inverse range is obtained by evaluating this function at energy $E=k^{2} / 2 \mu+i \epsilon$. The function $f_{X}(E)$ also gives the energy distribution from creation of the constituents at short distances. By the optical theorem, the distribution in the energy $E$ below the energy scale set by the range is proportional to the imaginary part of $f_{X}(E)$ :

$\operatorname{Im}\left[f_{X}(E+i \epsilon)\right]=\frac{\pi \gamma_{X}}{\mu} \delta\left(E+\gamma_{X}^{2} / 2 \mu\right)+\frac{\sqrt{2 \mu E}}{\gamma_{X}^{2}+2 \mu E} \theta(E)$.

There is a delta-function term at a negative energy from the production of the weakly bound molecule and a thetafunction term with positive energy from the production of the constituents of the molecule.

If the $X(3872)$ is a weakly bound charm-meson molecule, its constituents are the superposition of charm mesons in Eq. (1). We denote the masses of the spin-0 charm mesons $D^{0}$ and $D^{+}$by $M_{0}$ and $M_{1}$, the masses of the spin-1 charm mesons $D^{* 0}$ and $D^{*+}$ by $M_{* 0}$ and $M_{* 1}$ and the masses of the pions $\pi^{0}$ and $\pi^{+}$by $m_{0}$ and $m_{1}$ (or collectively by $m_{\pi}$ ). The range of the interactions between the charm mesons is $1 / m_{\pi}$. The corresponding energy scale $m_{\pi}^{2} / 2 \mu$ is about $10 \mathrm{MeV}$. This is comparable to the energy of the $D^{*+} D^{-}$scattering threshold, which is $8.2 \mathrm{MeV}$ above the $D^{* 0} \bar{D}^{0}$ scattering threshold. The present value of the difference $E_{X}$ between the mass of the $X$ and the energy of the $D^{* 0} \bar{D}^{0}$ scattering threshold is [14]

$E_{X} \equiv M_{X}-\left(M_{* 0}+M_{0}\right)=(+0.01 \pm 0.18) \mathrm{MeV}$.

The central value in Eq. (5) corresponds to a charm-meson pair above the scattering threshold. The value lower by $1 \sigma$ corresponds to a bound state with binding energy $\left|E_{X}\right|=$ $0.17 \mathrm{MeV}$ and binding momentum $\gamma_{X}=18 \mathrm{MeV}$.

The universal results for the wave function for a nearthreshold S-wave bound state in Eq. (2) and the scattering amplitude for its constituents in Eq. (3) can be derived from a zero-range effective field theory (ZREFT) with a single scattering channel [28]. In the case of $X$, the constituents are the neutral charm mesons $D^{* 0}, \bar{D}^{* 0}, D^{0}$, and $\bar{D}^{0}$ [29]. This ZREFT describes explicitly the $D^{* 0} \bar{D}^{0}$ and $D^{0} \bar{D}^{* 0}$ components of the $X$ with energies sufficiently close to their scattering threshold. Its range of validity extends at most up to the $D^{*+} D^{-}$scattering threshold, which is higher in energy by $8.2 \mathrm{MeV}$ and corresponds to a relative momentum for $D^{* 0} \bar{D}^{0}$ of $126 \mathrm{MeV}$. This effective field theory does not describe explicitly the $D^{0} \bar{D}^{0} \pi^{0}$ component of the $X$, which can arise from the decays $D^{* 0} \rightarrow D^{0} \pi^{0}$ or $\bar{D}^{* 0} \rightarrow \bar{D}^{0} \pi^{0}$.

Fleming, Kusunoki, Mehen and van Kolck developed an effective field theory called XEFT that has a much greater range of validity, because it describes pion interactions explicitly [27]. It is an effective field theory for neutral and charged charm mesons $D^{*}, \bar{D}^{*}, D$, and $\bar{D}$ and for neutral and charged pions $\pi$. XEFT describes explicitly the $D^{*} \bar{D}$, $D \bar{D}^{*}$, and $\bar{D} D \pi$ components of the $X$. The region of validity of the original formulation of XEFT extends to about the minimum energy required to produce a $\rho$ meson. For a 
charm meson pair, the region of validity is below a relative momentum of about $1000 \mathrm{MeV}$. For a charm meson pair plus a pion, the region of validity of XEFT is also limited by the nonrelativistic approximation for the pion: the relative momentum of the pion must be less than about $m_{\pi} \approx 140 \mathrm{MeV}$. We refer to a pion with relative momentum of order $m_{\pi}$ or smaller as a soft pion.

A Galilean-invariant formulation of XEFT that exploits the approximate conservation of mass in the transitions $D^{*} \leftrightarrow D \pi$ was developed in Ref. [30]. In Galilean-invariant XEFT, the spin- 0 charm mesons $D^{0}$ and $D^{+}$have the same kinetic mass $M_{0}$, the spin- 1 charm mesons $D^{* 0}$ and $D^{*+}$ have the same kinetic mass $M_{0}+m_{0}$, and the pions $\pi^{0}$ and $\pi^{+}$have the same kinetic mass $m_{0}$. The difference between the physical mass and the kinetic mass of a particle is taken into account through its rest energy. Galilean invariance simplifies the ultraviolet divergences of XEFT. The pion number defined by the sum of the numbers of $D^{*}, \bar{D}^{*}$, and $\pi$ mesons is conserved in Galilean-invariant XEFT. The region of validity of Galilean-invariant XEFT extends up to about the minimum energy required to produce an additional pion, which is above the $D^{*} \bar{D}$ threshold by about $140 \mathrm{MeV}$. For a charm-meson pair, the region of validity extends to a relative momentum of about $500 \mathrm{MeV}$.
In Ref. [31], Braaten, Hammer, and Mehen pointed out that XEFT could also be applied to sectors with pion number larger than 1 . In particular, it can be applied to the sector with pion number 2 , which consists of $D^{*} \bar{D}^{*}, D \bar{D}^{*} \pi$, $D^{*} \bar{D} \pi, D \bar{D} \pi \pi$, and $X \pi$. The cross sections for $D^{*} \bar{D}^{*} \rightarrow$ $D^{*} \bar{D}^{*}$ and $D^{*} \bar{D}^{*} \rightarrow X \pi$ at small kinetic energies were calculated in Ref. [31]. If a high energy process can create $D^{*} \bar{D}^{*}$ at short distances, XEFT can describe their subsequent rescattering into $X$ plus a soft pion. In Ref. [25], we applied XEFT to exclusive decays of $B$ mesons into $K X \pi$. In Sec. IV and Sec. V of this paper, we apply XEFT to the inclusive prompt production of $D^{*} \bar{D}^{*}$ and $X \pi$ with small relative momentum in high energy hadron collisions.

\section{PRODUCTION OF $X$}

If $X(3872)$ is a weakly bound charm-meson molecule with the flavor structure in Eq. (1), the cross section for producing $X$ can be expressed in terms of the amplitudes for producing $D^{* 0} \bar{D}^{0}$ and $D^{0} \bar{D}^{* 0}$ [22]. The inclusive prompt cross sections for producing $D^{* 0} \bar{D}^{0}$ with small relative momentum $\boldsymbol{k}$ in the charm-meson-pair rest frame and for producing $X$ can be expressed as

$$
\begin{aligned}
d \sigma\left[D^{* 0} \bar{D}^{0}\right] & =\frac{1}{\text { flux }} \sum_{y} \int d \Phi_{\left(D^{*} \bar{D}\right)+y}\left|\mathcal{A}_{D^{* 0} \bar{D}^{0}+y}(\boldsymbol{k})\right|^{2} \frac{d^{3} k}{(2 \pi)^{3} 2 \mu}, \\
d \sigma[X(3872)] & =\frac{1}{\text { flux }} \sum_{y} \int d \Phi_{\left(D^{*} \bar{D}\right)+y}\left|\int \frac{d^{3} k}{(2 \pi)^{3}} \psi_{X}(k) \frac{\mathcal{A}_{D^{* 0} \bar{D}^{0}+y}(\boldsymbol{k})+\mathcal{A}_{D^{0} \bar{D}^{* 0}+y}(\boldsymbol{k})}{\sqrt{2}}\right|^{2} \frac{1}{2 \mu},
\end{aligned}
$$

where $\mu$ is the reduced mass of $D^{* 0} \bar{D}^{0}$. The sums over $y$ are over all the additional particles that can be produced. The cross section for $D^{0} \bar{D}^{* 0}$ is the same as in Eq. (6a) with $\mathcal{A}_{D^{* 0} \bar{D}^{0}+y}(\boldsymbol{k})$ replaced by $\mathcal{A}_{D^{0} \bar{D}^{* 0}+y}(\boldsymbol{k})$. The chargeconjugation-even superposition of those amplitudes appears in the cross section for $X$ in Eq. (6b). The momentum-space wave function for the $X$ in Eq. (6b) is normalized so $\int\left(d^{3} k /(2 \pi)^{3}\right)\left|\psi_{X}(k)\right|^{2}=1$. The differential phase space $d \Phi_{\left(D^{*} \bar{D}\right)+y}$ is that for a composite particle denoted by $\left(D^{*} \bar{D}\right)$ with mass $M_{* 0}+M_{0}$ plus the additional particles $y$. Factors of 3 from the sums over the spin states of $D^{* 0}$ or $\bar{D}^{* 0}$ or $X$ are absorbed into the amplitudes $\mathcal{A}$. The phasespace integrals in Eqs. (6) are over the 3-momenta of the additional particles $y$, but the cross sections remain differential in the 3-momentum $\boldsymbol{P}$ of $\left(D^{*} \bar{D}\right)$. Thus the $D^{* 0} \bar{D}^{0}$ cross section in Eq. (6a) is differential in both $\boldsymbol{P}$ and $\boldsymbol{k}$, while the $X$ cross section in Eq. (6b) is differential only in $\boldsymbol{P}$.

Expressions for the cross sections in Eqs. (6) that take into account the $X$ resonance were presented in Ref. [23]. The cross sections in Eqs. (6) were expressed in factored forms, with long-distance factors that involve the binding momentum $\gamma_{X}$ and with short-distance factors that involve only momentum scales of order $m_{\pi}$ or larger. The amplitudes for producing $D^{* 0} \bar{D}^{0}+y$ in Eq. (6a) can be decomposed into charge-conjugation-even $(C=+)$ and charge-conjugation-odd $(C=-)$ components. The $C=+$ component is enhanced by the $X$ resonance. If the nonresonant $C=-$ component is neglected, the amplitude for producing $D^{* 0} \bar{D}^{0}+y$ can be expressed as a product of the $C=+$ component of a short-distance amplitude and a resonance factor that depends on $\gamma_{X}$ :

$$
\mathcal{A}_{D^{* 0} \bar{D}^{0}+y}(\boldsymbol{k})=\frac{1}{\sqrt{2}}\left(\frac{\mathcal{A}_{D^{* 0} \bar{D}^{0}+y}^{\text {s.d. }}+\mathcal{A}_{D^{0} \bar{D}^{* 0}+y}^{\text {s.d. }}}{\sqrt{2}}\right) \frac{\Lambda}{-\gamma_{X}-i k} .
$$

The expression for the corresponding amplitude $\mathcal{A}_{D^{0} \bar{D}^{* 0}+y}(\boldsymbol{k})$ is identical. The short-distance amplitudes $\mathcal{A}_{D^{* 0} \bar{D}^{0}+y}^{\text {s.d. }}$ and $\mathcal{A}_{D^{0} \bar{D}^{* 0}+y}^{\text {s.d. }}$ are independent of the momentum if $\boldsymbol{k}$ is small compared to $m_{\pi}$. The constant $\Lambda$ in the numerator of the resonance factor should be of order $m_{\pi}$. 
The only dependence on the small momentum $\gamma_{X}$ is in the denominator of the resonance factor. Since $\Lambda \gg \gamma_{X}$, the absolute value of the resonance factor is approximately 1 at $k=\Lambda$, so $\Lambda$ can be interpreted as the momentum scale where the amplitude becomes comparable in magnitude to the amplitude in the absence of the resonance.

The factorization formula for the $D^{* 0} \bar{D}^{0}$ cross section can be obtained simply by inserting the amplitude in Eq. (7) into Eq. (6a). The factorization formula for the $X$ cross section cannot be obtained so simply. If the universal wave function in Eq. (2) is inserted into Eq. (6b), the momentum integral is logarithmically ultraviolet divergent. The factorization formula for the $X$ cross section can be obtained instead by requiring the sum of the cross sections for producing $X$ and the cross sections for producing $D^{* 0} \bar{D}^{0}$ and $D^{0} \bar{D}^{* 0}$ integrated over $\boldsymbol{k}$ to be consistent with the optical theorem in Eq. (4). The resulting factorization formulas for the inclusive prompt cross sections are

$$
\begin{aligned}
d \sigma\left[D^{* 0} \bar{D}^{0}\right] & =\frac{1}{\text { flux }} \sum_{y} \int d \Phi_{\left(D^{*} \bar{D}\right)+y}\left|\mathcal{A}_{D^{D^{0}} \bar{D}^{0}+y}^{\text {s.d. }}+\mathcal{A}_{D^{0} \bar{D}^{* 0}+y}^{\text {s.d. }}\right|^{2} \frac{\Lambda^{2}}{\gamma_{X}^{2}+k^{2}} \frac{d^{3} k}{(2 \pi)^{3} 8 \mu}, \\
d \sigma[X(3872)] & =\frac{1}{\text { flux }} \sum_{y} \int d \Phi_{\left(D^{*} \bar{D}\right)+y}\left|\mathcal{A}_{D^{* 0} \bar{D}^{0}+y}^{\text {s.d. }}+\mathcal{A}_{D^{0} \bar{D}^{* 0}+y}^{\text {s.d. }}\right|^{2} \frac{\Lambda^{2} \gamma_{X}}{8 \pi \mu} .
\end{aligned}
$$

The differential cross section for $D^{* 0} \bar{D}^{0}$ in Eq. (8a) should be a good approximation up to relative momentum $k$ of about $\Lambda$.

In the expression for the $X$ cross section in Eq. (8b), there are interference terms between the short-distance amplitudes for producing $D^{* 0} \bar{D}^{0}+y$ and $D^{0} \bar{D}^{* 0}+y$. The interference terms are positive for some sets of additional final-state particles $y$ and negative for others. In highenergy hadron collisions, there are dozens or even hundreds of additional particles. The sum over the many additional particles $y$ gives cancellations that suppress the interference terms. The $X$ cross section in Eq. (6b) then reduces to the sum of a $D^{* 0} \bar{D}^{0}$ term and a $D^{0} \bar{D}^{* 0}$ term. At large transverse momentum, the hadronization of a $c \bar{c}$ pair is equally likely to produce $D^{* 0} \bar{D}^{0}$ and $D^{0} \bar{D}^{* 0}$, because the probability of a light quark or antiquark from a colliding hadron to become a constituent of one of the charm mesons is very small. The $D^{* 0} \bar{D}^{0}$ term and the $D^{0} \bar{D}^{* 0}$ term should therefore be equal, and the expression for the $X$ cross section reduces to

$d \sigma[X(3872)]=\frac{1}{\text { flux }} \sum_{y} \int d \Phi_{\left(D^{*} \bar{D}\right)+y}\left|\mathcal{A}_{D^{* 0} \bar{D}^{0}+y}^{\text {s.d }}\right|^{2} \frac{\Lambda^{2} \gamma_{X}}{4 \pi \mu}$.

\section{PRODUCTION OF A SPIN-1 CHARM-MESON PAIR}

XEFT can be applied to the production of $D^{*} \bar{D}^{*}$ through a short-distance process that creates the pair of charm mesons. A short-distance process is one in which all the other particles in the reaction have momenta in the $D^{*} \bar{D}^{*}$ center-of-momentum (CM) frame that are much larger than $m_{\pi}$. As far as the $D^{*}$ and $\bar{D}^{*}$ are concerned, the process can be described as a point interaction that creates $D^{*}$ and $\bar{D}^{*}$. The amplitude for producing $D^{*} \bar{D}^{*}$ can be represented in XEFT by the Feynman diagram in Fig. 1 with a vertex from which the $D^{*}$ and $\bar{D}^{*}$ emerge. We denote the vertex factor for the reaction that creates $D^{* 0} \bar{D}^{* 0}$ at a point and produces the set of additional particles $y$ by $i \mathcal{A}_{D^{* 0} \bar{D}^{* 0}+y}$, where $i$ and $j$ are the spin indices of the $D^{* 0}$ and $\bar{D}^{* 0}$. The matrix element for the reaction that produces $D^{* 0} \bar{D}^{* 0}$ with polarization vectors $\boldsymbol{\varepsilon}$ and $\overline{\boldsymbol{\varepsilon}}$ is $\mathcal{A}_{D^{* 0} \bar{D}^{* 0}+y}^{i j} \varepsilon^{i} \bar{\varepsilon}^{j}$.

To obtain the differential cross section for producing $D^{* 0} \bar{D}^{* 0}$, the amplitude $\mathcal{A}_{D^{* 0} \bar{D}^{* 0}+y}^{i j}$ must be multiplied by its complex conjugate $\left(\mathcal{A}_{D^{* 0} \bar{D}^{* 0}+y}\right)^{*}$. Their product must be summed over the additional particles $y$ (which includes sums over their spin states and integrals over their momenta), summed over the spin states of $D^{* 0}$ and $\bar{D}^{* 0}$, integrated over the phase space of $D^{* 0}$ and $\bar{D}^{* 0}$, and divided by the flux factor. The amplitude $\mathcal{A}_{D^{*} \bar{D}^{*}+y}^{i j}$ is a Cartesian tensor in the CM frame of $D^{*} \bar{D}^{*}$ with vector indices $i j$. It must be a linear combination of $\delta^{i j}$, terms of the form $\epsilon^{i j k} \hat{P}_{m}^{k}$ and $\hat{P}_{m}^{i} \hat{P}_{n}^{j}$ that involve unit vectors in the directions of the large momenta of some of the additional particles $y$, and terms that involve polarization vectors and tensors of the additional particles. For each set of additional

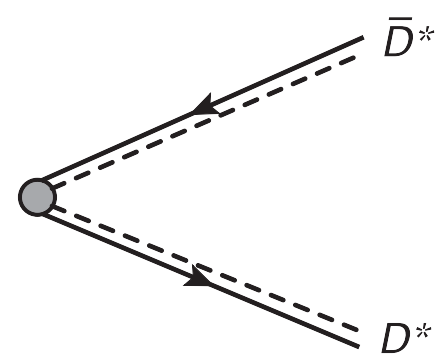

FIG. 1. Feynman diagram in XEFT for production of $D^{*} \bar{D}^{*}$ from their creation at a point. The $D^{*}$ and $\bar{D}^{*}$ are represented by double lines consisting of a dashed line and a solid line with an arrow. 
particles $y$, the product of $\mathcal{A}_{D^{*} \bar{D}^{*}+y}^{i j}$ and $\left(\mathcal{A}_{D^{*} \bar{D}^{*}+y}^{k l}\right)^{*}$ summed over their spins and integrated over their momenta is a 4-index Cartesian tensor that defines a density matrix in the spin indices $i j$ of the amplitude and the spin indices $k l$ of the complex conjugate amplitude. This tensor must be a linear combination of $\delta^{i j} \delta^{k l}$, $\delta^{i k} \delta^{j l}$, and $\delta^{i l} \delta^{j k}$. The term $\delta^{i k} \delta^{j l}$ is diagonal in the spin indices $i k$ of the charm meson and in the spin indices $j l$ of the anticharm meson. The terms $\delta^{i j} \delta^{k l}$ and $\delta^{i l} \delta^{j k}$ take into account entanglement between the spins. Their coefficients will be positive for some sets of the additional particles $y$ and negative for other sets. In the case of inclusive prompt production at the Tevatron or the LHC, cancellations from the sum over the many additional particles will suppress the $\delta^{i j} \delta^{k l}$ and $\delta^{i l} \delta^{j k}$ terms, leaving a density matrix proportional to $\delta^{i k} \delta^{j l}$. The weighted average from the sum over the additional particles $y$ therefore has the form

$$
\left\langle\mathcal{A}_{D^{* 0} \bar{D}^{* 0}+y}^{i j}\left(\mathcal{A}_{D^{* 0} \bar{D}^{* 0}+y}^{k l}\right)^{*}\right\rangle=\frac{1}{9}\left\langle\left|\mathcal{A}_{D^{* 0} \bar{D}^{* 0}+y}\right|^{2}\right\rangle \delta^{i k} \delta^{j l} .
$$

After multiplying by the polarization vectors, the sum over the spin states of $D^{* 0}$ and $\bar{D}^{* 0}$ gives

$\sum_{\text {spins }}\left\langle\mathcal{A}_{D^{* 0} \bar{D}^{* 0}+y}^{i j}\left(\mathcal{A}_{D^{* 0} \bar{D}^{* 0}+y}^{k l}\right)^{*}\right\rangle\left(\varepsilon^{i} \bar{\varepsilon}^{j}\right)\left(\varepsilon^{k} \bar{\varepsilon}^{l}\right)^{*}=\left\langle\left|\mathcal{A}_{D^{* 0} \bar{D}^{* 0}+y}\right|^{2}\right\rangle$.

The prefactor 1/9 in Eq. (10) was chosen so the prefactor in Eq. (11) would be 1.

The differential cross section for producing $D^{* 0} \bar{D}^{* 0}$ with large total momentum and small relative momentum $\boldsymbol{k}$ and with polarization vectors $\boldsymbol{\varepsilon}$ and $\overline{\boldsymbol{\varepsilon}}$ can be expressed as

$$
d \sigma\left[D^{* 0} \bar{D}^{* 0}\right]=\frac{1}{\text { flux }} \sum_{y} \int d \Phi_{\left(D^{*} \bar{D}^{*}\right)+y}\left|\mathcal{A}_{D^{* 0} \bar{D}^{* 0}+y}\right|^{2} \frac{d^{3} k}{(2 \pi)^{3} M_{* 0}} .
$$

The differential phase space $d \Phi_{\left(D^{*} \bar{D}^{*}\right)+y}$ is that for a composite particle denoted by $\left(D^{*} \bar{D}^{*}\right)$ with mass $2 M_{* 0}$ and the additional particles $y$. The integrals are over the 3 -momenta of the additional particles but not over the 3-momentum $\boldsymbol{P}$ of $\left(D^{*} \bar{D}^{*}\right)$. The cross section is therefore differential in $\boldsymbol{P}$ and $\boldsymbol{k}$. The sum over the spins of $D^{* 0}$ and $\bar{D}^{* 0}$ is implicit. We assume the $D^{* 0} \bar{D}^{* 0}$ channel has no resonant threshold enhancement analogous to that from the $X(3872)$ in the $D^{* 0} \bar{D}^{0}$ channel, so $\mathcal{A}_{D^{* 0} \bar{D}^{* 0}+y}^{i j}$ can be interpreted as a short-distance amplitude.

In the prompt production of a pair of charm mesons with large transverse momentum at a high-energy hadron collider, isospin symmetry and heavy-quark spin symmetry imply that the short-distance amplitudes for producing each pair of spin states for the charm mesons $D^{(*)} \bar{D}^{(*)}$ should be equal. Since we have absorbed factors of 3 from summing over spin states of $D^{*}$ and $\bar{D}^{*}$ into the amplitudes, the squared amplitude $\left|\mathcal{A}_{D^{* 0} \bar{D}^{* 0}+y}\right|^{2}$ in Eq. (12) should be equal to 3 times the corresponding squared short-distance amplitude $\left|\mathcal{A}_{D^{* 0} \bar{D}^{0}+y}^{\text {s.d }}\right|^{2}$ in the expression for the $X$ cross section in Eq. (9). For inclusive prompt production at the Tevatron or LHC, the total momentum of $D^{* 0} \bar{D}^{* 0}$ is large enough that the difference between the mass $2 M_{* 0}$ of $\left(D^{*} \bar{D}^{*}\right)$ and the mass $M_{* 0}+M_{0}$ of $\left(D^{*} \bar{D}\right)$ can be neglected in the phase space integrals. We can therefore eliminate the shortdistance factor in Eq. (12) in favor of the $X$ cross section in Eq. (9) at the expense of introducing the unknown momentum scale $\Lambda$ of order $m_{\pi}$ and the unknown binding momentum $\gamma_{X}$ :

$$
d \sigma\left[D^{* 0} \bar{D}^{* 0}\right] \approx d \sigma[X(3872)] \frac{12 \pi \mu}{\gamma_{X} \Lambda^{2}} \frac{d^{3} k}{(2 \pi)^{3} M_{* 0}} .
$$

\section{PRODUCTION OF $X \boldsymbol{\pi}$ NEAR $D^{*} \bar{D}^{*}$ THRESHOLD}

A pair of spin- 1 charm mesons $D^{* 0} \bar{D}^{* 0}$ created at short distances can rescatter into $X(3872) \pi^{0}$. The Feynman diagrams for $D^{* 0} \bar{D}^{* 0}$ created at a point to rescatter into $X \pi^{0}$ are shown in Fig. 2. The amplitude for these diagrams can be calculated using XEFT. In addition to the Feynman rules for Galilean-invariant XEFT given in [30], we need the vertices for the coupling of $D^{* 0} \bar{D}^{0}$ and $D^{0} \bar{D}^{* 0}$ to $X$. The vertices are given by $\left(\sqrt{\pi \gamma_{X}} / \mu\right) \delta^{i j}$, where $i$ and $j$ are the spin indices of the spin-1 charm meson and the $X$ [31]. If the amplitude for producing $D^{* 0} \bar{D}^{* 0}$ at short distances with specific polarization vectors is $\mathcal{A}_{D^{* 0} \bar{D}^{* 0}+y}^{i j} \varepsilon^{i} \bar{\varepsilon}^{j}$, the vertex for creating $D^{* 0} \bar{D}^{* 0}$ at a point in Fig. 2 is $i \mathcal{A}_{D^{* 0} \bar{D}^{* 0}+y}^{i j}$. That amplitude can be treated as a point vertex, because the momenta of the colliding hadrons and of the additional particles $y$ in the $X \pi$ rest frame are all large compared to the relative momentum $\boldsymbol{q}$ of $X$ and $\pi$. The integral over the loop energy is conveniently evaluated by contours using the pole of the propagator for the $D^{* 0}$ or $\bar{D}^{* 0}$ line attached to the $X$. The resulting expression for the amplitude for producing $X \pi^{0}$ with small relative momentum $\boldsymbol{q}$ in the $X \pi$ rest frame and with polarization vector $\boldsymbol{\varepsilon}$ for the $X$ is
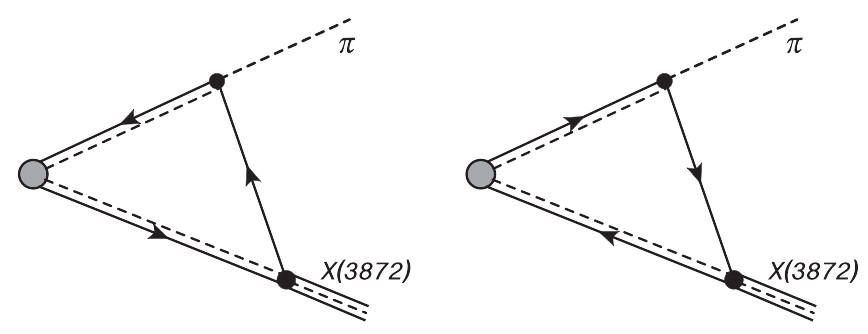

FIG. 2. Feynman diagrams in XEFT for $D^{* 0} \bar{D}^{* 0}$ created at a point to rescatter into $X \pi^{0}$. The $X(3872)$ is represented by a triple line with two solid lines and one dashed line. The $D$ and $\bar{D}$ are represented by solid lines. The $\pi$ is represented by a dashed line. 


$$
\begin{aligned}
& i \mathcal{A}_{D^{* 0} \bar{D}^{* 0}+y}^{i j} \frac{g\left(\pi \gamma_{X} / m_{0}\right)^{1 / 2}}{2 \mu f_{\pi}} \int \frac{d^{3} k}{(2 \pi)^{3}}\left[\frac{\left(M_{0}+m_{0}\right) q^{i}-m_{0} k^{i}}{M_{0}+m_{0}} \varepsilon^{j}+\varepsilon^{i} \frac{\left(M_{0}+m_{0}\right) q^{j}-m_{0} k^{j}}{M_{0}+m_{0}}\right] \\
& \quad \times \frac{1}{-\gamma_{X}^{2} /(2 \mu)+i \Gamma_{* 0} / 2-\mu\left((\boldsymbol{q}-\boldsymbol{k}) / M_{0}-\boldsymbol{k} /\left(M_{0}+m_{0}\right)\right)^{2} / 2} \\
& \quad \times \frac{1}{-\gamma_{X}^{2} /(2 \mu)-\delta_{0}+i \Gamma_{* 0}+\boldsymbol{q}^{2} /\left(2 \mu_{X \pi}\right)-\boldsymbol{k}^{2} /\left(M_{0}+m_{0}\right)},
\end{aligned}
$$

where $\mu=M_{0}\left(M_{0}+m_{0}\right) /\left(2 M_{0}+m_{0}\right)$ and $\mu_{X \pi}=\left(2 M_{0}+m_{0}\right) m_{0} /\left(2\left(M_{0}+m_{0}\right)\right)$ are the Galilean-invariant reduced masses of $D^{* 0} \bar{D}^{0}$ and $X \pi$. The integral is over the loop momentum $\boldsymbol{k}$ of the $D^{* 0}$ or $\bar{D}^{* 0}$ that becomes a constituent of the $X$. The remaining two propagators can be combined into a single denominator by introducing an integral over a Feynman parameter. The integral over the loop momentum can be evaluated analytically. The resulting amplitude for producing $X \pi^{0}$ is

$$
\begin{aligned}
& i \mathcal{A}_{D^{* 0} \bar{D}^{* 0}+y}^{i j} \frac{g\left(\pi \gamma_{X} / m_{0}\right)^{1 / 2} M_{* 0}^{3 / 2}}{16 \pi \mu f_{\pi}}\left(\varepsilon^{i} q^{j}+q^{i} \varepsilon^{j}\right) \int_{0}^{1} d x\left(\frac{2 M_{0}}{2 M_{0}+(1-x) m_{0}}\right)^{5 / 2} \\
& \quad \times\left[\left(\delta_{0}-\gamma_{X}^{2} / 2 \mu\right)-(1+x)\left(\delta_{0}-i \Gamma_{* 0} / 2\right)+\frac{x M_{0}}{\left(2 M_{0}+(1-x) m_{0}\right) \mu_{X \pi}} \boldsymbol{q}^{2}\right]^{-1 / 2},
\end{aligned}
$$

where $\delta_{0}=M_{* 0}-M_{0}-m_{0}=7.0 \mathrm{MeV}, \Gamma_{* 0} \approx 60 \mathrm{keV}$ is the predicted decay width of $D^{* 0}$ [30], and $g /\left(2 \sqrt{m_{0}} f_{\pi}\right)=$ $0.30 / m_{0}^{3 / 2}$ is the coupling constant for the pion-emission vertex [30]. The final integral over $x$ can also be evaluated analytically if the integrand is simplified using $m_{0} \ll M_{0}$. This reduces the prefactor of $\boldsymbol{q}^{2}$ in the denominator of the integrand to $x / 2 m_{0}$. Our final result for the amplitude is rather simple:

$$
i \mathcal{A}_{D^{* 0} \bar{D}^{* 0}+y}^{i j} \frac{g\left(\pi \gamma_{X} M_{* 0}^{3} / m_{0}\right)^{1 / 2}}{8 \pi \mu f_{\pi}} \frac{\varepsilon^{i} q^{j}+q^{i} \varepsilon^{j}}{\sqrt{q^{2} / 2 m_{0}-\delta_{0}-\gamma_{X}^{2} / 2 \mu+i \Gamma_{* 0}}+\sqrt{-\gamma_{X}^{2} / 2 \mu+i \Gamma_{* 0} / 2}} .
$$

The denominator is a kinematic singularity factor that would have a zero at $q^{2}=2 m_{0} \delta_{0}$ if the binding momentum $\gamma_{X}$ and the width $\Gamma_{* 0}$ were both zero. The kinematic singularity is called a triangle singularity, because it arises from the region where the three charm-meson lines that form a triangle in the Feynman diagrams in Fig. 2 are all simultaneously on shell.

A pair of spin- 1 charm mesons $D^{*+} \bar{D}^{* 0}$ created at short distances can rescatter into $X \pi^{+}$. The Feynman diagram is the second diagram in Fig. 2, with the virtual $D^{* 0}$ line replaced by a $D^{*+}$ line and the final-state $\pi^{0}$ replaced by $\pi^{+}$. The integral over the loop momentum can be evaluated analytically. The final integral over $x$ can also be evaluated analytically if the integrand is simplified using $m_{0} \ll M_{0}$. Our final result for the amplitude is rather simple:

$$
i \mathcal{A}_{D^{*+} \bar{D}^{* 0}+y}^{i j} \frac{g\left(2 \pi \gamma_{X} M_{* 0}^{3} / m_{0}\right)^{1 / 2}}{8 \pi \mu f_{\pi}} \times \frac{q^{i} \varepsilon^{j}}{\sqrt{q^{2} / 2 m_{0}-\delta_{1}-\gamma_{X}^{2} / 2 \mu+i\left(\Gamma_{* 0}+\Gamma_{* 1}\right) / 2}+\sqrt{-\gamma_{X}^{2} / 2 \mu+i \Gamma_{* 0} / 2}}
$$

where $\delta_{1}=M_{* 1}-M_{0}-m_{1}=5.9 \mathrm{MeV} \quad$ and $\quad \Gamma_{* 1} \approx$ $83 \mathrm{keV}$ is the measured decay width of $D^{*+}$. The pair of spin- 1 charm mesons $D^{* 0} D^{*-}$ created at short distances can rescatter into $X \pi^{-}$. The amplitude is the same as that in Eq. (17) except that the short-distance factor is replaced by $\mathcal{A}_{D^{* 0} D^{*-}+y}$ and $q^{i} \varepsilon^{j}$ is replaced by $\varepsilon^{i} q^{j}$. The denominator of Eq. (17) is a triangle-singularity factor that would have a zero at $q^{2}=2 m_{0} \delta_{1}$ if the binding momentum $\gamma_{X}$ and the widths $\Gamma_{* 0}$ and $\Gamma_{* 1}$ were all zero.
Our expressions for the amplitudes in Eqs. (16) and (17) should be accurate provided the momentum integral in Eq. (14) is dominated by regions where the relative momentum $\boldsymbol{k}$ of the charm mesons that form the $X$ is less than about $m_{\pi}$. This condition imposes a constraint on the relative momentum $\boldsymbol{q}$ of $X$ and $\pi$. The constraint can be deduced from the integrated expression in Eq. (15) by requiring the energy proportional to $\boldsymbol{q}^{2}$ inside the last factor to be less than $m_{\pi}^{2} / 2 \mu$. At $x=1$, this energy is 
$E_{X \pi}=q^{2} / 2 \mu_{X \pi}$. Thus the kinetic energy $E_{X \pi}$ must be less than about $m_{\pi}^{2} / 2 \mu \approx 10 \mathrm{MeV}$.

To obtain the differential cross section for producing $X \pi^{0}$ and $X \pi^{+}$, the amplitudes in Eqs. (16) and (17) must be multiplied by their complex conjugates. Their product must be summed over the additional particles $y$ (which includes sums over their spin states and integrals over their momenta), summed over the spin states of $X$, integrated over the phase space of $X$ and $\pi$, and divided by the flux factor. For each set of additional particles $y$, the product of
$\mathcal{A}_{D^{*} \bar{D}^{*}+y}^{i j}$ and $\left(\mathcal{A}_{D^{*} \bar{D}^{*}+y}^{k l}\right)^{*}$ summed over their spins and integrated over their momenta, is a density matrix in the spin indices $i j$ and $k l$. In the case of inclusive prompt production at the Tevatron or the LHC, cancellations from the sum over the many additional particles $y$ leaves a density matrix diagonal in the indices $i k$ and in the indices $j l$, as in Eq. (10). After multiplying by the tensors in Eqs. (16) and (17) that involve polarization vectors, the sum over the spin states of $X$ results in a factor $\boldsymbol{q}^{2}$ :

$$
\begin{gathered}
\sum_{\text {spins }}\left\langle\mathcal{A}_{D^{* 0} \bar{D}^{* 0}+y}^{i j}\left(\mathcal{A}_{D^{* 0} \bar{D}^{* 0}+y}^{k l}\right)^{*}\right\rangle\left(\varepsilon^{i} q^{j}+q^{i} \mathcal{\varepsilon}^{j}\right)\left(\varepsilon^{k} q^{l}+q^{k} \varepsilon^{l}\right)^{*}=\frac{8}{9}\left\langle\left|\mathcal{A}_{D^{* 0} \bar{D}^{* 0}+y}\right|^{2}\right\rangle \boldsymbol{q}^{2}, \\
\sum_{\text {spins }}\left\langle\mathcal{A}_{D^{*+} \bar{D}^{* 0}+y}^{i j}\left(\mathcal{A}_{D^{*+} \bar{D}^{* 0}+y}^{k l}\right)^{*}\right\rangle\left(q^{i} \varepsilon^{j}\right)\left(q^{k} \varepsilon^{l}\right)^{*}=\frac{1}{3}\left\langle\left|\mathcal{A}_{D^{*+} \bar{D}^{* 0}+y}\right|^{2}\right\rangle \boldsymbol{q}^{2} .
\end{gathered}
$$

The squared amplitude $\left|\mathcal{A}_{D^{* 0} \bar{D}^{* 0}+y}\right|^{2}$ in Eq. (18a) also appears in the short-distance factor in the cross section for producing $D^{* 0} \bar{D}^{* 0}$ in Eq. (12). The short-distance factor in the cross section for producing $X \pi^{0}$ can therefore be eliminated in favor of the differential cross section for producing $D^{* 0} \bar{D}^{* 0}$ near the threshold given by Eq. (12). Alternatively the short-distance factor can be eliminated in favor of the cross section for producing $X$ by using Eq. (13).

The differential cross sections for producing $X \pi^{0}$ and $X \pi^{+}$with small relative momentum $\boldsymbol{q}$ can be expressed as

$$
\begin{aligned}
& \frac{d \sigma}{d^{3} q}\left[X \pi^{0}\right]=\sigma[X] \frac{g^{2} M_{* 0}^{3}}{48 \pi^{3} \mu \mu_{X \pi} f_{\pi}^{2} \Lambda^{2}} \frac{q^{2} / 2 m_{0}}{\left|\sqrt{q^{2} / 2 m_{0}-\delta_{0}-\gamma_{X}^{2} / 2 \mu+i \Gamma_{* 0}}+\sqrt{-\gamma_{X}^{2} / 2 \mu+i \Gamma_{* 0} / 2}\right|^{2}}, \\
& \frac{d \sigma}{d^{3} q}\left[X \pi^{+}\right]=\sigma[X] \frac{g^{2} M_{* 0}^{3}}{64 \pi^{3} \mu \mu_{X \pi} f_{\pi}^{2} \Lambda^{2}} \frac{q^{2} / 2 m_{0}}{\left|\sqrt{q^{2} / 2 m_{0}-\delta_{1}-\gamma_{X}^{2} / 2 \mu+i\left(\Gamma_{* 0}+\Gamma_{* 1}\right) / 2}+\sqrt{-\gamma_{X}^{2} / 2 \mu+i \Gamma_{* 0} / 2}\right|^{2}}
\end{aligned}
$$

We have used Eq. (13) to eliminate the short-distance factors in favor of the cross section for producing $X$ at the expense of introducing the unknown momentum scale $\Lambda$ of order $m_{\pi}$. The factors of the unknown binding momentum $\gamma_{X}$ have canceled. The differential cross section for producing $X \pi^{-}$is also given by Eq. (19b).

A convenient kinematic variable to consider is the kinetic energy $E_{X \pi}=q^{2} / 2 \mu_{X \pi}$ of $X \pi$ in the $X \pi$ CM frame, since the square of the $X \pi$ invariant mass near the $X \pi$ threshold is approximately linear in $E_{X \pi}$. The dependence of the differential cross sections $d \sigma / d E_{X \pi}$ on $E_{X \pi}$ is illustrated in Fig. 3 for $\gamma_{X}=18 \mathrm{MeV}$. The differential cross section for $X \pi^{0}$ in Fig. 3 has a narrow peak in $E_{X \pi}$ near $\delta_{0}=7.0 \mathrm{MeV}$ that comes from a charm-meson triangle singularity. The full width at half maximum of the denominator factor in Eq. (19a) is approximately $1.17 \gamma_{X}^{2} / 2 \mu$ if the binding energy $\gamma_{X}^{2} / 2 \mu$ is large compared to $\Gamma_{* 0}$ and approximately $6.21 \Gamma_{* 0} \approx 370 \mathrm{keV}$ if $\gamma_{X}^{2} / 2 \mu$ is small compared to $\Gamma_{* 0}$. The differential cross section for $X \pi^{+}$in Fig. 3 has a narrow peak in $E_{X \pi}$ near $\delta_{1}=5.9 \mathrm{MeV}$ that comes from a charm- meson triangle singularity. The full width at half maximum of the denominator factor in Eq. (19b) is approximately $1.17 \gamma_{X}^{2} / 2 \mu$ if $\gamma_{X}^{2} / 2 \mu$ is large compared to $\Gamma_{* 0}$ and $\Gamma_{* 1}$ and approximately $430 \mathrm{keV}$ if $\gamma_{X}^{2} / 2 \mu$ is small compared to $\Gamma_{* 0}$ and $\Gamma_{* 1}$.

The sensitivity of the narrow peaks in Fig. 3 to the binding energy $\left|E_{X}\right|=\gamma_{X}^{2} / 2 \mu$ is illustrated in Fig. 4. The differential cross section $d \sigma / d E_{X \pi}$ has a factor of the square root of $\left|E_{X}\right|$ that is removed by dividing by $\sigma[X]$. If $\left|E_{X}\right|$ is decreased from $0.17 \mathrm{MeV}$ to 0 , the height of the peak increases by about a factor of 2 . If $\left|E_{X}\right|$ is increased from $0.17 \mathrm{MeV}$ to $0.34 \mathrm{MeV}$, the height of the peak decreases by about a factor of 0.7 . The areas under the peaks are less sensitive to the binding energy.

The contributions of the triangle singularities to the integrated cross sections for $X \pi$ can be estimated by integrating the momentum distributions in Eqs. (19) from the threshold to some energy $E_{\max }$ beyond the peak. In the limits $\Gamma_{* 0} \rightarrow 0$ and $\gamma_{X} \ll \sqrt{\mu \delta_{0}}$, the integral of the momentum dependent factor in Eq. (19a) over the region $|\boldsymbol{q}|<q_{\max }$ is 


$$
\begin{aligned}
& \int_{q<q_{\max }} \frac{d^{3} q}{(2 \pi)^{3}} \frac{q^{2} / 2 m_{0}}{\left|\sqrt{q^{2} / 2 m_{0}-\delta_{0}-\gamma_{X}^{2} / 2 \mu+i \epsilon}+i \sqrt{\gamma_{X}^{2} / 2 \mu}\right|^{2}} \\
& \quad=\frac{1}{2 \pi^{2}}\left(2 m_{0} \delta_{0}\right)^{3 / 2} \times\left[\log \frac{8 \mu \delta_{0}}{\gamma_{X}^{2}}+\frac{1}{3}\left(\frac{q_{\max }^{2}}{2 m_{0} \delta_{0}}\right)^{3 / 2}+\left(\frac{q_{\max }^{2}}{2 m_{0} \delta_{0}}\right)^{1 / 2}-\frac{1}{2} \log \frac{\sqrt{q_{\max }^{2} / 2 m_{0} \delta_{0}}+1}{\sqrt{q_{\max }^{2} / 2 m_{0} \delta_{0}}-1}-\frac{11}{3}\right] .
\end{aligned}
$$

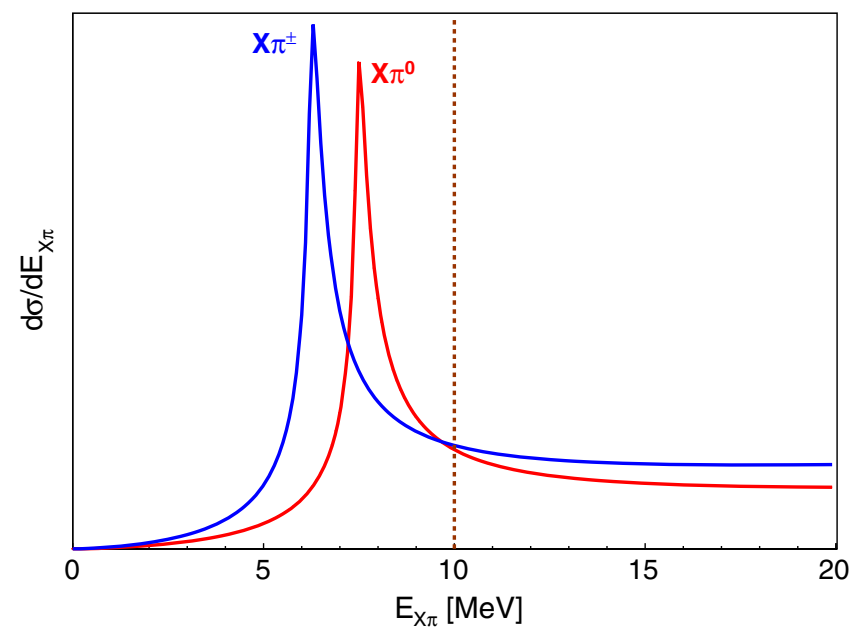

FIG. 3. Differential cross sections $d \sigma / d E_{X \pi}$ as functions of the kinetic energy $E_{X \pi}$ for $X \pi^{0}$ and for $X \pi^{ \pm}$summed over \pm from rescattering of $D^{*} \bar{D}^{*}$ created at short distances. The binding energy of the $X$ is $0.17 \mathrm{MeV}$. The region of validity of XEFT extends out to about the vertical dotted line at $E_{X \pi}=10 \mathrm{MeV}$. The scale on the vertical axis is arbitrary, but the relative normalization of the two curves is determined.

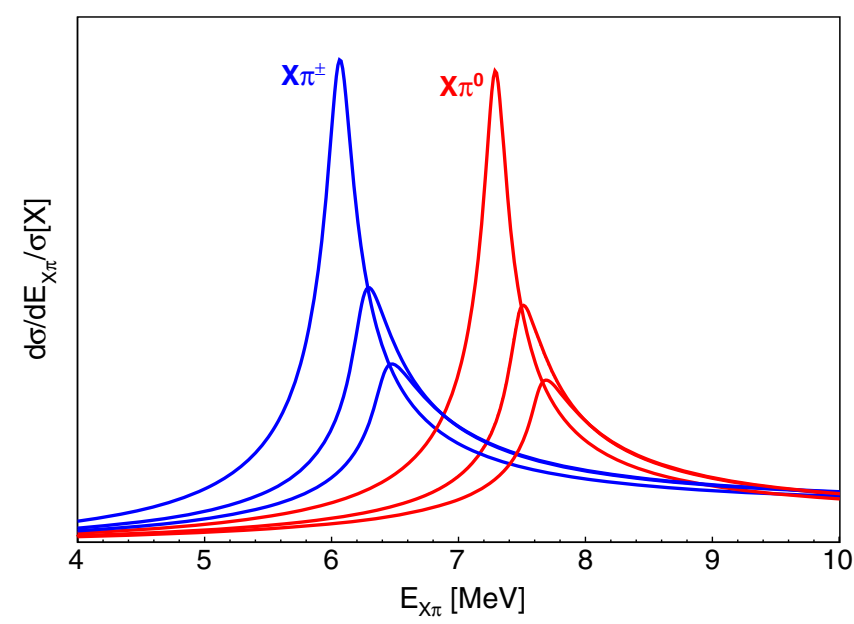

FIG. 4. Differential cross sections $d \sigma / d E_{X \pi} / \sigma[X]$ as functions of the kinetic energy $E_{X \pi}$ for $X \pi^{0}$ (right curves) and for $X \pi^{ \pm}$ summed over \pm (left curves). In order of decreasing height, the curves are for binding energies $E_{X}=0,\left|E_{X}\right|=0.17 \mathrm{MeV}$, and $\left|E_{X}\right|=0.34 \mathrm{MeV}$. The scale on the vertical axis is arbitrary, but the relative normalizations of the curves are determined.
The coefficient of $\left(2 m_{0} \delta_{0}\right)^{3 / 2}$ diverges logarithmically as $\gamma_{X} \rightarrow 0$. If we do not take the limit $\Gamma_{* 0} \rightarrow 0$, the coefficient of $\left(m_{0} \delta_{0}\right)^{3 / 2}$ also depends logarithmically on $\Gamma_{* 0}$. In the limit $\Gamma_{* 0} \rightarrow 0$ and $\Gamma_{* 1} \rightarrow 0$, the integral of the momentum dependent factor in Eq. (19b) is given by Eq. (20) with $\delta_{0}$ replaced by $\delta_{1}$.

We can use our expressions for the differential cross sections for $X \pi$ in Eq. (19) to estimate the cross sections for producing $X \pi$ in the peak from the triangle singularity. We denote the region of the peak by $(X \pi)_{\Delta}$. We declare that region to be $E_{X \pi}$ from 0 up to $E_{\max }=2 \delta_{0}$ for $\left(X \pi^{0}\right)_{\Delta}$ and up to $E_{\max }=2 \delta_{1}$ for $\left(X \pi^{ \pm}\right)_{\Delta}$. We approximate the integrals over the momentum distributions in Eqs. (19a) and (19b) using the integral in Eq. (20) and the analogous integral with $\delta_{0}$ replaced by $\delta_{1}$. The resulting estimates of the ratios of the cross sections for $(X \pi)_{\Delta}$ to the cross section for $X$ as a function of the binding energy $\left|E_{X}\right|=\gamma_{X}^{2} / 2 \mu$ is

$$
\begin{aligned}
& \frac{\sigma\left[\left(X \pi^{0}\right)_{\Delta}\right]}{\sigma[X]} \approx 0.049\left(\frac{m_{\pi}}{\Lambda}\right)^{2}\left[2.82-\log \frac{\left|E_{X}\right|}{0.17 \mathrm{MeV}}\right], \\
& \frac{\sigma\left[\left(X \pi^{+}\right)_{\Delta}\right]}{\sigma[X]} \approx 0.028\left(\frac{m_{\pi}}{\Lambda}\right)^{2}\left[2.64-\log \frac{\left|E_{X}\right|}{0.17 \mathrm{MeV}}\right] .
\end{aligned}
$$

The prefactors were obtained by setting $m_{\pi}=m_{0}$. The cross section for producing $\left(X \pi^{-}\right)_{\Delta}$ should be the same as that for producing $\left(X \pi^{+}\right)_{\Delta}$. If the binding energy of $X$ is not too far from $0.17 \mathrm{MeV}$, the ratio of the cross section for producing $\left(X \pi^{ \pm}\right)_{\Delta}$ summed over \pm to the cross section for producing $X$ without an accompanying pion is predicted to be $0.14\left(m_{\pi} / \Lambda\right)^{2}$. It is only logarithmically sensitive to the binding energy $\left|E_{X}\right|$, but it depends strongly on the unknown momentum scale $\Lambda$ of order $m_{\pi}$.

The inclusive prompt production of $X$ in $p p$ collisions has been studied at the Large Hadron Collider (LHC) by the CMS [20] and ATLAS [21] collaborations. The inclusive production of $X$ in $p p$ collisions has also been studied at the LHC by the LHCb [19], but they did not separate the prompt production from production from decay of bottom hadrons. The CMS collaboration measured the $\pi^{+} \pi^{-}$ invariant mass distribution from the decays of $X$ into $J / \psi \pi^{+} \pi^{-}[20]$. They reported the $X$ yield in the appropriate kinematic region to be $6302 \pm 346$ events. If the binding energy of $X$ is $0.17 \mathrm{MeV}$, the yield in $\left(X \pi^{ \pm}\right)_{\Delta}$ summed over \pm is predicted by Eq. (21b) to be smaller by the factor $0.14\left(m_{\pi} / \Lambda\right)^{2}$. Despite the uncertainty from the unknown scale $\Lambda$, this is large enough to encourage a search for the 
peak from the charm-meson triangle singularity at the LHC.

\section{SUMMARY AND DISCUSSION}

We have discussed the inclusive prompt production of the $X(3872)$ at high-energy hadron colliders under the assumption that the $X$ is a weakly bound charm-meson molecule with the particle content in Eq. (1). We considered the production of $X$ through the creation of a charm-meson pair at short distances of order $1 / m_{\pi}$ or smaller. The formation of the $X$ proceeds on longer distance scales, and it can be described by the effective field theory XEFT. The $X$ can be produced by the creation of its constituents $D^{* 0} \bar{D}^{0}$ and $D^{0} \bar{D}^{* 0}$ at short distances followed by the binding of the charm mesons into $X$. The $X$ can also be produced by the creation of a pair of spin- 1 charm mesons $D^{*} \bar{D}^{*}$ at short distances followed by the rescattering of the charm mesons into $X \pi$.

The differential cross sections for the inclusive prompt production of $X \pi^{0}$ from rescattering of $D^{* 0} \bar{D}^{* 0}$ and of $X \pi^{+}$ from rescattering of $D^{*+} \bar{D}^{* 0}$ are given in Eqs. (19) and illustrated in Fig. 4. They have a narrow peak from a charmmeson triangle singularity at a kinetic energy $E_{X \pi}$ close to the $D^{* 0} \bar{D}^{* 0}$ and $D^{*+} \bar{D}^{* 0}$ scattering thresholds, respectively. Estimates of the ratio of the cross sections for producing $X \pi^{0}$ and $X \pi^{ \pm}$in the peak from the charm-meson triangle singularity to the cross section for producing $X$ without an accompanying pion are given in Eqs. (21).
The prompt production of $X$ accompanied by $\pi^{+}$or $\pi^{-}$ can be studied at a hadron collider. The charged pion provides a clean signature for this new production mechanism. For prompt production at the LHC, the observation of this signature may be complicated by the combinatorial background from the hundreds of pions produced at the primary interaction vertex. The combinatorial background is especially severe in forward production, which is measured by the LHCb detector. For central production at large transverse momentum, which is measured by the ATLAS and CMS detectors, the combinatorial background may be more manageable. Given the number of $X$ events that have been observed, the number of events in the peak in the $X \pi^{ \pm}$invariant mass distribution from the charm-meson triangle singularity can be estimated using Eq. (21b). The estimate is large enough to encourage the effort to observe this peak at the LHC. The observation of such a peak would provide strong support for the identification of $X$ as a weakly bound charm-meson molecule and present a serious challenge to other models.

\section{ACKNOWLEDGMENTS}

This work was supported in part by the Department of Energy under Grant No. DE-SC0011726 and by the National Science Foundation under Grant No. PHY1607190. We thank T. Skwarnicki for valuable comments.
[1] H. X. Chen, W. Chen, X. Liu, and S. L. Zhu, The hidden-charm pentaquark and tetraquark states, Phys. Rep. 639, 1 (2016).

[2] A. Hosaka, T. Iijima, K. Miyabayashi, Y. Sakai, and S. Yasui, Exotic hadrons with heavy flavors: $X, Y, Z$, and related states, Prog. Theor. Exp. Phys. 2016, 062 C01 (2016).

[3] R. F. Lebed, R. E. Mitchell, and E. S. Swanson, Heavyquark QCD exotica, Prog. Part. Nucl. Phys. 93, 143 (2017).

[4] A. Esposito, A. Pilloni, and A. D. Polosa, Multiquark resonances, Phys. Rep. 668, 1 (2017).

[5] F. K. Guo, C. Hanhart, U. G. Meißner, Q. Wang, Q. Zhao, and B. S. Zou, Hadronic molecules, Rev. Mod. Phys. 90, 015004 (2018).

[6] A. Ali, J. S. Lange, and S. Stone, Exotics: Heavy pentaquarks and tetraquarks, Prog. Part. Nucl. Phys. 97, 123 (2017).

[7] S. L. Olsen, T. Skwarnicki, and D. Zieminska, Nonstandard heavy mesons and baryons: Experimental evidence, Rev. Mod. Phys. 90, 015003 (2018).

[8] M. Karliner, J. L. Rosner, and T. Skwarnicki, Multiquark states, Annu. Rev. Nucl. Part. Sci. 68, 17 (2018).

[9] C. Z. Yuan, The $X Y Z$ states revisited, Int. J. Mod. Phys. A 33, 1830018 (2018).
[10] N. Brambilla, S. Eidelman, C. Hanhart, A. Nefediev, C. P. Shen, C. E. Thomas, A. Vairo, and C. Z. Yuan, The $X Y Z$ states: Experimental and theoretical status and perspectives, arXiv:1907.07583.

[11] S. K. Choi et al. (Belle Collaboration), Observation of a Narrow Charmonium-Like State in Exclusive $B^{ \pm} \rightarrow$ $K^{ \pm} \pi^{+} \pi^{-} J / \psi$ Decays, Phys. Rev. Lett. 91, 262001 (2003).

[12] D. Acosta et al. (CDF Collaboration), Observation of the Narrow State $X(3872) \rightarrow J / \psi \pi^{+} \pi^{-}$in $\bar{p} p$ Collisions at $\sqrt{s}=1.96 \mathrm{TeV}$, Phys. Rev. Lett. 93, 072001 (2004).

[13] R. Aaij et al. (LHCb Collaboration), Determination of the $X(3872)$ Meson Quantum Numbers, Phys. Rev. Lett. 110, 222001 (2013).

[14] M. Tanabashi et al. (Particle Data Group), Review of particle physics, Phys. Rev. D 98, 030001 (2018).

[15] F. E. Close and P. R. Page, The $D^{* 0} \bar{D}^{0}$ threshold resonance, Phys. Lett. B 578, 119 (2004).

[16] M. B. Voloshin, Interference and binding effects in decays of possible molecular component of $X(3872)$, Phys. Lett. B 579, 316 (2004). 
[17] M. Ablikim et al. (BESIII Collaboration), Observation of the Decay $X(3872) \rightarrow \pi^{0} \chi_{c 1}(1 P)$, Phys. Rev. Lett. 122, 202001 (2019).

[18] V. M. Abazov et al. (D0 Collaboration), Observation and Properties of the $X(3872)$ Decaying to $J / \psi \pi^{+} \pi^{-}$in $p \bar{p}$ Collisions at $\sqrt{s}=1.96 \mathrm{TeV}$, Phys. Rev. Lett. 93, 162002 (2004).

[19] R. Aaij et al. (LHCb Collaboration), Observation of $X(3872)$ production in $p p$ collisions at $\sqrt{s}=7 \mathrm{TeV}$, Eur. Phys. J. C 72, 1972 (2012).

[20] S. Chatrchyan et al. (CMS Collaboration), Measurement of the $X(3872)$ production cross section via decays to $J / \psi \pi \pi$ in $p p$ collisions at $\sqrt{s}=7 \mathrm{TeV}, \mathrm{J}$. High Energy Phys. 04 (2013) 154.

[21] M. Aaboud et al. (ATLAS Collaboration), Measurements of $\psi(2 S)$ and $X(3872) \rightarrow J / \psi \pi^{+} \pi^{-}$production in $p p$ collisions at $\sqrt{s}=8 \mathrm{TeV}$ with the ATLAS detector, J. High Energy Phys. 01 (2017) 117.

[22] C. Bignamini, B. Grinstein, F. Piccinini, A. D. Polosa, and C. Sabelli, Is the $X(3872)$ Production Cross Section at Tevatron Compatible with a Hadron Molecule Interpretation? Phys. Rev. Lett. 103, 162001 (2009).

[23] P. Artoisenet and E. Braaten, Production of the $X(3872)$ at the tevatron and the LHC, Phys. Rev. D 81, 114018 (2010).
[24] E. Braaten, L.-P. He, and K. Ingles, Estimates of the $X(3872)$ cross section at a hadron collider, arXiv: 1811.08876.

[25] E. Braaten, L.-P. He, and K. Ingles, Production of $X(3872)$ Accompanied by a pion in $B$ meson decay, Phys. Rev. D 100, 074028 (2019).

[26] V. Poireau and M. Zito, A precise isospin analysis of $B \rightarrow \bar{D}^{(*)} D^{(*)} K$ decays, Phys. Lett. B 704, 559 (2011).

[27] S. Fleming, M. Kusunoki, T. Mehen, and U. van Kolck, Pion interactions in the $X(3872)$, Phys. Rev. D 76, 034006 (2007).

[28] E. Braaten and H.-W. Hammer, Universality in few-body systems with large scattering length, Phys. Rep. 428, 259 (2006).

[29] E. Braaten and M. Kusunoki, Factorization in the production and decay of the $X(3872)$, Phys. Rev. D 72, 014012 (2005).

[30] E. Braaten, Galilean-invariant effective field theory for the X(3872), Phys. Rev. D 91, 114007 (2015).

[31] E. Braaten, H.-W. Hammer, and T. Mehen, Scattering of an ultrasoft pion and the $X(3872)$, Phys. Rev. D 82, 034018 (2010). 